Topiques, études satoriennes

Topoï Studies, Journal of the SATOR

\title{
Maître-disciple : une relation topique
}

\section{Véronique Duché, Madeleine Jeay and Yen-Mai Tran-Gervat}

Volume 4, 2018

Maître-disciple : une relation topique

URI: https://id.erudit.org/iderudit/1074715ar

DOI: https://doi.org/10.7202/1074715ar

See table of contents

Publisher(s)

SATOR, Société d'Analyse de la Topique Romanesque d'Ancien Régime

ISSN

2369-4831 (digital)

Explore this journal

Cite this document

Duché, V., Jeay, M. \& Tran-Gervat, Y.-M. (2018). Maître-disciple : une relation topique. Topiques, études satoriennes / Topoï Studies, Journal of the SATOR, 4, 1-9. https://doi.org/10.7202/1074715ar
Article abstract

Si c'est au personnage de Fénelon que le mentor doit son nom - Mentor est le précepteur du héros éponyme des Aventures de Télémaque (1699) - le mentorat, ce lien qui, selon Françoise Waquet « signifie à la fois l'autorité et l'affection, la filiation et la continuité des générations, le don des connaissances et la production des talents», existe depuis bien plus longtemps. Il nous a semblé intéressant d'étudier dans la durée ce lien à la fois émotif, intellectuel et social, et d'en examiner l'expression dans les textes d'Ancien Régime tout particulièrement.

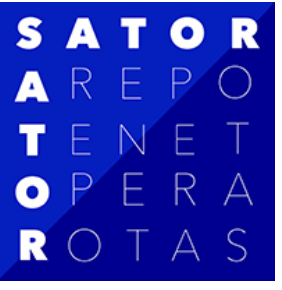

Tous droits réservés (c) Véronique Duché, Madeleine Jeay, Yen-Mai Tran-Gervat, 2019

This document is protected by copyright law. Use of the services of Érudit (including reproduction) is subject to its terms and conditions, which can be viewed online.

https://apropos.erudit.org/en/users/policy-on-use/ 


\section{Maître-disciple : une relation topique}

Si c'est au personnage de Fénelon que le mentor doit son nom - Mentor est le précepteur du héros éponyme des Aventures de Télémaque (1699) - le mentorat, ce lien qui, selon Françoise Waquet «signifie à la fois l'autorité et l'affection, la filiation et la continuité des générations, le don des connaissances et la production des talents ${ }^{1} »$, existe depuis bien plus longtemps. Il nous a semblé intéressant d'étudier dans la durée ce lien à la fois émotif, intellectuel et social, et d'en examiner l'expression dans les textes d'Ancien Régime tout particulièrement. Dans la lignée des travaux de F. Waquet, notamment Les Enfants de Socrate. Généalogie intellectuelle et transmission du savoir, XVIIe-XXIe siècles (2008), il s'agira ici de s'interroger sur la question de la transmission et de l'apprentissage, selon l'angle de l'analyse topique qui fait le propre des travaux conduits depuis plusieurs années par la SATOR. Toutefois la relation de mentorat ne sera pas restreinte à la seule relation maître-disciple et à ses tropes, pour embrasser d'autres formes de transmission et d'apprentissage.

La relation complexe du maître avec son disciple fait l'objet d'une longue tradition de recherche. Deux volumes collectifs récents - De l'un à l'autre. Maîtres et disciples $(2013)^{2}$ et Figures du maître. De l'autorité à l'autonomie $(2013)^{3}$ - ont récemment relancé l'intérêt pour ce champ, où George Steiner ${ }^{4}$ et Francis Wolff ${ }^{5}$ apparaissent comme les références contemporaines majeures des études menées sur la question. Ce volume vise à élargir l'enquête en s'intéressant à d'autres aspects du mentorat. Un bref parcours chronologique permettra de mieux situer les dix études qui suivent.

$\mathrm{Au}$ commencement était Homère. Apparu dans l'Odyssée déjà, Mentor est « un des plus fidelles amis d'Ulysse, et celui à qui, en s'embarquant pour Troye, il avoit confié le soin de toute sa maison afin qu'il la conduisît sous les ordres du bon Laërte $»^{6}$. Ce sage conseiller est en réalité la déesse Athéna déguisée, qui se rend à Ithaque «pour exciter son fils [Télémaque] et pour lui inspirer la force dont il a besoin, afin qu'appellant les Grecs à une Assemblée, il ait le courage de s'opposer à l'insolence des Princes qui poursuivent sa mere, et qui égorgent continuellement ses bœufs et ses moutons pour faire des sacrifices et des festins. $»^{7} \mathrm{C}^{\prime}$ est

1 Françoise Waquet, Les enfants de Socrate. Filiation intellectuelle et transmission du savoir XVIIe- XXIe siècle, Paris, Albin Michel, 2008, p. 310.

2 Aurélie Névot (dir.), De l'un à l'autre. Maîtres et disciples, CNRS éditions, Paris, 2013.

${ }^{3}$ Cristina Noacco, Corinne Bonnet, Patrick Marot et Charalampos Orfanos (dir.), Figures du maître. De

l'autorité à l'autonomie, Presses Universitaires de Rennes, coll. «Interférence », 2013.

${ }^{4}$ George Steiner, Maîtres et disciples. Paris, Éditions Gallimard, Folio essais, 2003.

[Lessons of the Masters, Cambridge, Mass. : Harvard University Press, c2003.]

${ }^{5}$ Francis Wolff, L'être, l'homme, le disciple. Figures philosophiques empruntées aux Anciens, P.U.F., coll. « Quadrige », 2000.

${ }^{6}$ Homère, L'Odyssée, Chant I, Livre II, Traduction Madame Dacier, Amsterdam, Wetsteins \& Smith, 1731, p. 84.

7 Homère, L'Odyssée, Chant I, Livre I, op. cit., p. 24-25.

https://journals.uvic.ca/index.php/sator/index 
Athéna qui guide Télémaque jusqu'à Pylos, puis lui conseille de rentrer à Ithaque (Chant 15) et lui indique comment déjouer le piège des prétendants. Toutefois, selon Madame Dacier ${ }^{8}$, la traductrice du XVIII ${ }^{\mathrm{e}}$ siècle, Mentor renvoie à une personne « réelle », proche de l'auteur :

Ce Mentor est un des amis d'Homere qui l'a placé ici par reconnaissance, parce qu'étant abordé à Ithaque à son retour d'Espagne, et se trouvant fort incommodé d'une fluxion sur les yeux, qui l'empêcha de continuer son voyage, il fut reçû chez ce Mentor, qui eut de lui tous les soins imaginables.

Homère établit donc le prototype du bon mentor, celui qui cumule les fonctions de manuductor ${ }^{9}$ («celui qui conduit par la main» selon Albert le Grand ${ }^{10}$ ) et de Vir bonus, «homme digne d'admiration non pas pour son érudition, mais pour les effets qu'elle produit $»^{11}$.

La relation pédagogique qui lie un disciple à son maître a été abondamment explorée par les écrivains et romanciers depuis Homère ${ }^{12}$. Le bon maitre s'affirme ainsi comme celui qui, bien que dans une posture magistrale, "élève » son disciple. Il est un "Auctor », au double sens de porteur de l'auctoritas, et de celui qui augmente, accroît (augere) les facultés de son disciple.

Et c'est Socrate bien sûr qui incarne la figure du parfait mentor ${ }^{13}$. Selon F. Waquet, « cette figure est celle du maître qui n'impose pas son savoir, voire celui qui, dans son inscience, interroge sans affirmer et qui, en pratiquant, par le dialogue, une sorte d'accouchement, amène le disciple à trouver le savoir qu'il a en lui. ${ }^{14}$

À cet archétype du bon maître, qui vise à l'autonomie de ses disciples et qui les aide «à devenir eux-mêmes $\rangle^{15}$, s'oppose le maître à penser qui s'impose à son élève.

Les exemples abondent de ces mauvais maîtres. Ainsi Lucien de Samosate (vers 120 - après 180) dans son Biôn prasis, ou Vitarum auctio, met en scène une foire aux maîtres ${ }^{16}$, dans laquelle il ne se prive pas de faire la satire des mauvais pédagogues. ${ }^{17}$

\footnotetext{
${ }^{8}$ Homère, L'Odyssée, op. cit., note 60, p. 84.

${ }^{9}$ Chez Fénelon, Mentor guide Télémaque « par la main » (Fénelon, Les Aventures de Télémaque, ed. J.-L. Goré, Garnier-Flammarion, 1969, p. 504).

${ }^{10}$ Albertus Magnus, Super Dionysium De divinibus nominibus, c. 2, n. 87 (cité par Julie Casteigt, « Comment passer de l'ignorance à la connaissance ? Une figure du maître manuductor selon Albert le Grand », in Figures du maître. De l'autorité à l'autonomie, op. cit., p. 51.

${ }^{11}$ Nicolas Corréard, «Satire des maîtres et mise en questions de l'idéal humaniste du Quattrocento dans les imitations de la Vitarum Auctio de Lucien de Samosate (Vies de philosophes à vendre) », in Figures du maître. De l'autorité à l'autonomie, op. cit., p. 202.

${ }^{12}$ Le topos satorien correspondant pourra être formulé ainsi : Relation éducation maître disciple.

${ }^{13}$ Dans son « defilé des icônes », outre Socrate, F. Waquet fait notamment prendre place Tycho Brahé, le Christ, Confucius, Ser Brunetto, le docteur Faust (p. 12-13).

${ }^{14}$ Françoise Waquet, Les enfants de Socrate, op. cit., p. 75-76.

15 Idem, p. 256.

${ }^{16}$ L'ouvrage a été traduit en français sous divers titres : Les sectes à l'encan, Philosophes à vendre, ou encore, tout récemment, Vies de philosophes à vendre.

17 Voir à ce sujet Nicolas Corréard, «La foire aux maîtres : l'imitation de la Vitarum auctio de Lucien de Samosate dans le contexte de la Renaissance érasmienne, évangélique et sceptique », Anabases, 15, 2012, p. 8799.
}

https://journals.uvic.ca/index.php/sator/index 
Jérôme (v. 347-419/420), amené à côtoyer des maîtres aussi divers que célèbres (dont le grammairien Donat, ou le Père cappadocien Grégoire de Naziance), n'est pas en reste et dans ses écrits dénonce les maîtres flatteurs et hypocrites, les censeurs et les incapables ${ }^{18}$. Le moine de Bethléem distingue dans son Commentaire sur l'Epître aux Galates quatre catégories magistrales :

On observe la quadruple division suivante : celui qui enseigne remplit son office, mais stérile est l'auditeur; ou bien l'auditeur est d'un bon naturel mais, par l'inhabileté de celui qui enseigne, la semence de la parole meurt; ou bien, à la vérité, celui qui est enseigné est aussi insensé que celui qui instruit; rarement, il arrive que maître et disciple s'accordent, c'est-à-dire que celui qui enseigne autant que celui-là peut absorber ou que celui qui est enseigné peut accueillir autant que celui qui enseigne peut donner ${ }^{19}$.

La critique Morgane Kieffer insiste sur la dimension collective de la transmission envisagée dans une perspective hyéronimienne :

elle doit se faire dans l'ordre d'une chaîne chronologique et pédagogique qui implique une éthique de la communauté : on n'apprend pas pour soi seul, c'est aux autres que l'on doit d'apprendre. Jérôme s'inscrit dans la tradition initiée par Longin, de l'émulation et de l'imitation : l'aemulatio, élan inspiré par les grands maîtres, mène au sublime par l'imitatio de cet exemple. ${ }^{20}$

Mais le mentor n'est pas seulement un maître en Écritures saintes. La transmission intellectuelle peut s'enrichir de notes affectives. Le mentor se fait passeur de savoir-faire comme de savoir-vivre. Ainsi au Moyen Âge, la littérature courtoise abonde en figures de jeunes héros dont les "enfances » sont guidées par un écuyer dévoué, comme Gouvernal auprès de Tristan. ${ }^{21}$

C'est à la figure érémitique, prise dans sa dimension pédagogique, que s'intéresse tout particulièrement Paul Bretel dans sa belle étude sur Les ermites et les moines dans la littérature française $d u$ Moyen $\hat{A g} e^{22}$. Il rappelle qu'un jeune frère pouvait rejoindre un groupe d'ermites, « à condition qu'il s'installât auprès d'un ancien qui lui servirait de maître,

\footnotetext{
18 « Il y a les maîtres flatteurs, dont les paroles ne servent à rien, les censeurs, qui critiquent au lieu de corriger, les précepteurs hypocrites qui ne vivent pas selon leurs enseignements, ceux qui ne croient pas en ce qu'ils enseignent, ceux qui ont une vie exemplaire mais qui sont incapables d'enseigner à leurs disciples ce qu'ils vivent, faute de connaissances suffisantes, ceux qui se laissent aller à la colère quand c'est le calme qui sied à un maître. » (Régis Coutray, « La figure du maître chez Jérôme. La science des écritures ou la clé du royaume », in Figures du maitre, op. cit., p. 17).

${ }^{19}$ Commentaire sur l'Epître aux Galates, 2,4,19. (Cité par R. Coutray, art. cit., p. 18) Préciser de qui est la traduction?

${ }^{20}$ Morgane Kieffer, « Maître \& disciple : les relais du savoir des premiers théoriciens à nos jours », Acta fabula, vol. 14, $\mathrm{n}^{\circ}$ 6, Essais critiques, Septembre 2013, URL : http://www.fabula.org/lodel/acta/index.php?id=8047, page consultée le 03 octobre 2017.

${ }^{21}$ Jacques Verger, Des nains sur des épaules de géants, Paris, Taillandier, 2006, p. 160.

${ }^{22}$ Paul Bretel, Les ermites et les moines dans la littérature française du Moyen Âge. 1150-1250, Paris, Champion, 1995.
} 
comme cela se pratiquait, aux origines du monachisme, dans les déserts orientaux $»^{23}$, alors que dans le Coenobium, les postulants étaient pris en charge par un préposé aux novices. Selon P. Bretel, « le recours au père spirituel, dont on sollicite les conseils ou une parole de sagesse, est un lieu commun dans la littérature des Vies des Pères du désert et dans les Apophtegmes $\gg{ }^{24}$ Le critique en relève des exemples dans la Continuation de Manessier, comme dans celle de Gerbert de Montreuil ou dans le Lancelot en prose, le Livre d'Artus présentant pour sa part «l'une des plus remarquables propédeutiques à la vie érémitique assurées par un ancien $»{ }^{25}$

Et Bretel d'ébaucher le portrait de ces mentors, ou maistres :

Sont dit[s] maistres des ermites qui sont certes des lettrés, c'est-à-dire [...] qui ont une culture profane et qui, surtout, connaissent les Écritures ; mais leur clergie, solidaire de leur sainteté et de la dignité que leur confère leur âge, leur permet d'exercer sur leurs compagnons et sur leurs visiteurs, une autorité aussi bien temporelle que morale et spirituelle. ${ }^{26}$

L'ermite au Moyen Âge joue le rôle non seulement de guide moral ou spirituel ${ }^{27}$ mais également de conseiller politique. «Le roman arthurien offre ainsi des exemples de rois conseillés par des moines : Léodegan, père de Guenièvre, raconte ainsi à Merlin que c'est sur les conseils d'un ermite qu'il a renoncé à compléter la Table Ronde, laissant cinquante sièges vacants sur les cent cinquante disponibles ». ${ }^{28}$ On pourra relever de nombreux épisodes dans le Lancelot, comme celui où "un prudons de grant savoir " vient donner une "leçon de politique royale » à Arthur ${ }^{29}$, ou encore celui où Amustan se charge de convertir les mœurs d'Arthur dans l'épisode de la fausse Guenièvre. L'ermite parfois n'hésite pas à s'écarter d'une interprétation stricte de la loi, comme en témoigne le rôle ambigu joué par Ogrin dans le Tristan de Béroul, qui à deux reprises se fait le complice des amants.

La chanson de geste accorde également à l'ermite le rôle de conseiller spirituel. Girart de Roussillon, chanson hagiographique, détaille l'itinéraire pénitentiel du héros. P. Bretel souligne le relief singulier pris par Berthe, l'épouse de Girart, qui « se tien[t] constamment aux côtés de son époux dans l'apprentissage qu'il fait du renoncement [...] et de l'humilité » et veille « par ses encouragements et par son exemple $»^{30}$ à ce que le héros s'amende.

On pourra s'arrêter sur le texte essentiel que constitue le Conte du Graal de Chrétien de Troyes. Dans ce roman initiatique, Perceval reçoit une triple éducation - chevaleresque, courtoise et religieuse - prodiguée successivement par plusieurs mentors. Élevé à l'écart de la cour, dans l'univers sauvage de la forêt, le jeune «nice », décidé à devenir un chevalier,

\footnotetext{
${ }^{23}$ Paul Bretel, op. cit., p. 151.

${ }^{24}$ P. Bretel, op. cit., p. 152.

${ }^{25}$ P. Bretel, op. cit., p. 154.

${ }^{26}$ P. Bretel, op. cit., p. 157.

${ }^{27}$ Le topos satorien correspondant pourra être formulé ainsi : Guider_conduite morale. Dans le cas d'un mentor relevant du domaine sacré, le topos pourra être modulé ainsi : relation_figure sacrée_disciple.

${ }^{28}$ P. Bretel, op. cit., p. 653.

29 T. 8, XLIX.

${ }^{30}$ P. Bretel, op. cit., p. 606.
}

https://journals.uvic.ca/index.php/sator/index 
reçoit les «chastoiements" de sa mère, mais ne les comprend pas $^{31}$. C'est le vavasseur Gornemant de Goort qui lui permettra à la fois de découvrir les secrets de la chevalerie, et de faire siennes les leçons maternelles. D'autres rencontres (Blanchefleur, Gauvain) seront nécessaires avant que le jeune Gallois n'achève son parcours et devienne un chevalier accompli. Ainsi c'est l'oncle et ermite qui se charge du volet religieux de cette éducation, permettant à un Perceval en détresse morale et spirituelle d'accéder à la chevalerie célestielle. Selon P. Bretel, « on s'accorde généralement à voir dans la rencontre de Perceval avec son oncle l'ermite $[\ldots]$ le prototype d'un motif littéraire promis à une extraordinaire fortune $»^{32}$.

Ainsi le roman de chevalerie de la Renaissance recourt encore à ce motif. Au Second livre de Amadis de Gaule, Andahod «le sainct Hermite», recueille un Amadis désespéré qu'il nomme « Beau Tenebreux » :

Mon filz vous estes jeune, \& de belle taille : ce nonobstant vostre vie est tenebreuse pour vostre enny, pourtant je veulx que vous soyez nommé beau Tenebreux : ce que Amadis eut pour aggreable $\left[\ldots . .{ }^{33}\right.$

Amadis fait pénitence auprès de lui en son ermitage situé «en un lieu desert [...] dans la mer», et appelé la Roche pauvre «pour la sterilité du lieu » ${ }^{34}$. Lorsqu'Amadis, enfin pardonné par Oriane, quitte l'ermitage, il assure à Andahod :

jour de ma vie ne mettray en oubly le bien que vous m'avez fait, car sans vous je feusse mort et de corps et d'ame : [...] par voz devotes prieres (comme je croy) il a pleu à dieu me conserver, et donner vie jusques icy. ${ }^{35}$

D'autres membres de la famille d'Amadis ont également affaire à des ermites qui les conseillent et les guident. Ainsi au Premier Livre de Amadis de Gaule, Galaor, le second fils de Périon et Élisenne, est enlevé par un géant et confié à « un Hermite de tressaincte vie ", chargé de le «nourrir » et de lui enseigner « tout ce qui est convenable à Chevalier »

Quant à Esplandian, le fils d'Amadis, il est guidé par un ermite « laïc » (ce dernier a une fille) au chapitre 3 du Cinqiesme livre d'Amadis de Gaule, qui le recueille et le soigne après son combat contre les trois chevaliers géants.

$\mathrm{Au} \mathrm{XVII}{ }^{\mathrm{e}}$ siècle encore, le motif de la rencontre avec l'ermite se lit sous la plume d'Honoré d'Urfé, décliné toutefois sous sa forme païenne. En effet c'est le grand Druide Adamas qui aide Céladon en lui conseillant de se déguiser en druidesse et de fréquenter Astrée sous le nom d'Alexis ${ }^{37}$.

\footnotetext{
${ }^{31}$ Chrétien de Troyes, Perceval ou le Conte du Graal, éd. J. Dufournet, Paris, GF Flammarion, 1997, v. $525-$ 596.

${ }^{32}$ P. Bretel, op. cit., p. 607. Voir p. 611-616 l'analyse de cet épisode crucial.

${ }^{33}$ Le Second Livre de Amadis de Gaule, Paris, Denis Janot, 1541, fo XIX.

${ }^{34}$ Amadis de Gaule. Livre II, $\mathrm{f}^{\circ}$ XIX-XIXv.

${ }^{35}$ Amadis de Gaule. Livre II, fo XXXIII.

${ }^{36}$ G. Rodríguez de Montalvo, Amadis de Gaule. Livre I, trad. Herberay des Essarts, éd. M. Bideaux, Paris, Honoré Champion, 2006, p. 221.

${ }^{37}$ Honoré d'Urfé, L'Astrée, Livre III.
} 
Outre les ermites, d'autres types de mentors se rencontrent toutefois dans la littérature française comme européenne. Ainsi chez Dante, c'est Virgile qui joue le rôle de mentor et conduit le narrateur à travers l'enfer et le purgatoire, alors que Béatrice prendra le relais pour le guider au paradis, tandis que Christine de Pizan les prend tous deux comme guides dans Le Livre du chemin de long estude ${ }^{38}$.

La fée Urgande la descogneue, qui apparaît dès le chapitre III du Premier Livre d'Amadis de Gaule peut également être retenue parmi les avatars de Mentor. À la fois adjuvant - elle apporte une lance à Amadis (chap. 6), le délivre des sortilèges d'Arcalaus (chap. 20) - elle est également celle qui préside à la destinée du héros et balise son chemin par ses prophéties. C'est avec elle que Gandales, qui a recueilli le Demoysel de la Mer alors abandonné par Élisenne, désirerait conférer «des affaires de cest enfant ${ }^{39}$. Sa présence se fera sentir tout au long des cinq premiers livres de Montalvo. On rappellera ainsi que le mentorat n'est pas genré, et que des personnages féminins peuvent jouer le rôle de guide moral et social ${ }^{40}$. C'est ce que s'est attachée à démontrer Christine de Pizan en élisant d'abord des figures féminines - la Sibylle de Cumes, les allégories Raison, Droiture et Justice, la déesse Othéa - puis en assumant elle-même cette position (article de M. Jeay). X. Bonnier montre dans ce volume comment la Délie de Maurice Scève est pour lui une nouvelle Diotime qui lui apprend à aimer et à haïr, qui l'initie aux véritables valeurs de l'amour, comme Laure pour Pétrarque. Quant aux auteurs femmes de contes de fées présentées par M. Legault, elles s'adressent à leur public féminin sans doute en guides morales, mais surtout en facteurs d'émancipation, les accompagnant dans la recherche d'un cheminement personnel. Toutefois, note F. Waquet dans l'ouvrage cité, le mot mentor n'existe pas au féminin : « mentrix ne s'utilise pas $»^{41}$.

L'humanisme et son intérêt pour l'éducation stimulent la plume des écrivains de la Renaissance. Si l'éducateur, le maître, est bien présent chez Rabelais - on pense bien sûr à Ponocrates, qui institue Gargantua «en telle discipline, qu'il ne perdoit heure du jour $»^{42}-$, d'autres personnages jouent le rôle de mentor: Eudemon auprès du jeune Gargantua, ou Pantagruel lui-même auprès de Panurge lorsque ce dernier «se conseille à Pantagruel pour sçavoir s'il se doibt marier $»^{43}$. Fénelon pour sa part réactive le prototype homérien dans ses Aventures de Télémaque ${ }^{44}$. Rousseau lui emboîtera le pas avec Emile, ou de l'Education. Sophie, qui a lu Fénelon, considère Émile comme son Télémaque. Le tuteur pour sa part affirme : «Émile n'étant pas roi, ni moi dieu, nous ne tourmentons point de ne pouvoir imiter Télémaque et Mentor $»^{45}$.

\footnotetext{
${ }^{38}$ Le topos satorien correspondant pourra être formulé ainsi : Guider_voyage_initiatique. On notera que Dante évoque également la figure de Brunetto Latini, philosophe et orateur, qui l'encouragea sur la voie des études (Dante, L'Enfer, Chant XV, v. 82-87). Au sujet de la figure du maître chez Dante, voir G. Lombardo, « La figure du maître dans la Divine Comédie de Dante », in Figures du maître, op. cit., p. 97-112.

39 Amadis de Gaule, Livre I, p. 210.

${ }^{40}$ Le topos satorien correspondant pourra être formulé ainsi : Femme_exercer_rôle de mentor.

${ }^{41}$ F. Waquet, p. 289.

${ }^{42}$ F. Rabelais, Gargantua, chapitre 21 (1535).

${ }^{43}$ F. Rabelais, Tiers Livre, chapitre IX.

${ }^{44}$ Voir à ce sujet J.-Ph. Grosperrin, « Le maître, le père, l'ami. Pédagogie et fantasme dans les fictions de Fénelon », in Figures du maître, op. cit., p. 185-198.

${ }^{45}$ Rousseau, Emile, ou de l'Education, Paris, Garnier-Flammarion, 1966, p. 612.
} 
L'imitatio se révèle être un concept-clef pour définir la relation entre le mentor et son protégé. Ainsi de Vasari, explorant cette relation dans les domaines artistique et littéraire dans ses Vies des plus excellents peintres, sculpteurs, et architectes $(1550)^{46}$. La posture d'autorité constitutive de la fonction pédagogique au sens large du terme peut se développer en amitié. Cette dimension affective colore le lien que Clément Marot entretien avec son père Jean, maître en poésie, mais aussi guide bienveillant, " plus souvent père que poète », selon E. Delvallée dans son article.

Mais ce processus n'est pas sans danger, pour le disciple comme pour le maître. Il en est ainsi lorsque la relation devient trop personnelle - l'on pense par exemple à Héloïse et Abélard ${ }^{47}$. Ce paradoxe de la séduction et l'ambiguïté de la figure magistrale, analysée par Steiner, se lisent également sous la plume de Fénelon, où Mentor, selon Benedetta Papàsogli, est à la fois «humain et divin, masculin et féminin, vieux et jeune, lieu de réconciliation des contraires $»^{48}$. Chez Balzac, la relation qui lie Lucien de Rubempré à l'Abbé Herrera (Vautrin) se révèle ambiguë ${ }^{49}$.

Il reste que les mauvais mentors se trouvent à foison dans la littérature d'Ancien Régime. Outre les « precepteurs sorbonagres » de Gargantua, on trouve des mentors qui, bien que se revendiquant de Fénelon, ne sont pas au niveau. Ainsi du Chevalier de Rasac dans Le mentor cavalier : ou, Les illustres infortunez de notre siècle, du marquis d'Argens ${ }^{50}$ :

Un jeune Homme, appellé le Comte de Beauval, dont le Cœur est naturellement porté à la Tendresse, choisit pour Compagnon de Voïage le Chevalier de Rasac, d'un Age àpeu-près semblable au sien ; mais, aussi en garde contre ses Passions, qu'il s'y livre luimême aisément. Les Conseils de Rasac, Le Mentor de cet Ouvrage, ramenent enfin à la Raison et à la Tranquillité le Comte de Beauval, qu'on en peut considérer comme le Telemaque. ${ }^{51}$

Ou encore de Madame de Merteuil qui, dans Les Liaisons Dangereuses désire « faire des éducations » et exerce sa mauvaise influence sur Cécile comme sur Danceny. Parmi les mauvais mentors, figure aussi le type du maître tyran représenté sous la figure du régent qui se promène le fouet à la main ( $\mathrm{P}$. Gautier), jouissant du contrôle qu'il peut exercer. Ce contrôle peut passer, comme dans Le Doyen de Prévost analysé ici par A. Cheng, par la manipulation afin de triompher des disciples mais aussi des autres éducateurs perçus comme des rivaux. Appartient également à cette catégorie celui qui transmet un savoir sans valeur issu de lectures mal assimilées, comme le Don Quichotte présenté par Y.-M. Tran-Gervat, qui se croit cependant détenteur de vérité. C'est cette sorte de maître caricatural qu'évoque le

\footnotetext{
${ }^{46}$ Voir à ce sujet I. Cotensin, « Maître et disciple dans les recueils de Vies d'artistes (Vasari et ses successeurs romains du XVII ${ }^{\mathrm{e}}$ siècle », in Figures du maître, op. cit., p. 297-308.

${ }^{47}$ Voir à ce sujet G. Lobrichon, ou, Les illustres « La dialectique du maître et du disciple : Abélard et Héloïse », in Figures du maître, op. cit., p. 171-183.

${ }^{48}$ Benedetta Papàsogli, « Mentor souriant », Revue italienne d'études françaises, 42014.

${ }^{49}$ Balzac, Splendeurs et misères des courtisanes. Voir à ce sujet Priscilla P. Clark, « The metamorphoses of Mentor : Fénelon to Balzac », Romanic Review, 75 (2), 1984, p. 200-215.

${ }^{50}$ Jean-Baptiste de Boyer, Le mentor cavalier : ou, Les illustres infortunez de notre siècle, Londres, Aux Dépens de la Compagnie, 1736.

${ }^{51}$ Idem, Avertissement, np.
} 
Pangloss du Candide de Voltaire qui dépossède de son sens le discours philosophique (S. Viselli). Mais la figure la plus pernicieuse, celle qu'on pourrait qualifier d'anti-mentor, est le libertin qui initie son disciple à des valeurs et des comportements qui s'opposent à la morale traditionnelle, comme le guide que rencontre le Francion de Sorel au cours de ses aventures et dans Les Lettres du marquis de Roselle de Madame Elie de Beaumont, le jeune marquis pris dans les filets du libertin Valville, prototype de ceux qui foisonnent dans la fiction du XVIII ${ }^{\mathrm{e}}$ siècle.

L'échec de la relation de mentorat peut venir aussi du mentoré, de sa résistance aux leçons du mentor. La jeune princesse du Livre des trois Vertus de Christine de Pizan va à l'encontre des conseils de prudence de sa gouvernante et s'abandonne aux plaisirs de l'amour courtois (M. Jeay). C'est de la même façon l'emportement de la jeunesse qui fait obstacle à la sagesse que l'oncle veut inculquer à son élève dans Le Télémaque travesti de Marivaux, comme chez Madame de Tencin, la puissance des élans du cœur et des illusions de la passion.

La finalité du processus d'apprentissage, que celui-ci soit de nature pratique, dans le cas du peintre d'enluminures étudié par C. Brunon, ou qu'il implique l'acquisition de compétences sociales, intellectuelles ou morales, est le renversement de la relation entre mentor et mentoré, quand le disciple devient lui-même mentor. C'est tout le sens du parcours que met en scène Christine de Pizan, d'abord guidée par les personnages allégoriques qui lui servent d'alter ego, puis prenant son indépendance par rapport à elles et parlant en son nom propre pour s'adresser aux figures de pouvoir qu'elle estime devoir conseiller. À propos du Francion de Sorel, P. Gauthier montre comment, à l'issue du roman, l'expérience que le héros a acquise lui permet d'être en mesure de délivrer lui-même des leçons de vie. C'est aussi ce qui semblerait se jouer entre Don Quichotte et Sancho Panza si le renversement d'autorité entre le maître et le valet ne relevait pas plutôt du changement ponctuel des rôles, «parenthèse carnavalesque» dans ce qui est de l'ordre de la parodie de la relation de mentorat (Y.-M. Tran-Gervat). Cette redistribution des rôles se joue aussi dans Jacques le Fataliste où celui qui a besoin d'être instruit, ce n'est pas le valet, mais le maître, remise en question du principe d'autorité normalement assumé par le mentor.

Dans ces œuvres à portée comique, s'opère un renversement de la relation de mentorat qui sollicite la complicité du lecteur et, par la même occasion, l'invite à une prise de distance réflexive quant au fondement même de la notion d'autorité. Cette remise en cause d'une vision traditionnelle du mentorat a certes précédé celle que s'attache à déconstruire Diderot dont le Neveu de Rameau met en lumière « les apories de la transmission ». Cependant avec lui naîtrait « une figure moderne du modèle saisi dans sa vérité humaine et non plus dans son statut d'exemple institué et 'non négociable'» (M.-G. Gouverneur). Il y aurait donc une dimension historique attachée au personnage du mentor, un point tournant de sa représentation étant d'après $\mathrm{S}$. Viselli, le XVIII siècle qui est le témoin d'une véritable métamorphose dans la mesure où il tend à perdre sa dimension allégorique pour s'incarner et faire ressortir ce que peut avoir d'ambigu la relation de mentorat.

Si elle est avérée, cette évolution ne peut que renvoyer à la situation en miroir d'auteurs ayant acquis une portée allégorique pour incarner une image de guide dans les œuvres de leurs successeurs, par exemple Virgile pour Dante, eux-mêmes repris, avec Boèce, par Christine de Pizan. C'est aussi ce que laisse entendre, en dépit de sa dénégation - « Marot je suis, \& Maro ne suis pas »-, l'homonymie qu'exhibe Clément Marot avec le nom de Virgile, s'inscrivant 
lui aussi dans sa lignée, point initial d'une filiation qui passe par Jean, le père (E. Delvallée) pour aboutir à lui-même assumant la position de mentor. Ou bien Maurice Scève, lorsqu'il identifie sa Délie avec Diotime, parmi une série de personnages féminins évoquant le désir, pour montrer que leur mentorat ne fait que servir d'alibi à sa propre affirmation de soi dans ce rôle. En définitive, faire de soi un mentor, n'est-ce pas l'aspiration de tout auteur ? Cela peut passer par les jalons que sont de prestigieux modèles, comme nous venons de le voir, ou de façon avouée chez les conteuses de contes de fées. Quel que soit le cas, il s'agit d'une ambition qui renvoie à la responsabilité de l'auteur et pose la question posée par Cervantès de la validité du mentorat livresque ou par Prévost lorsqu'il appuie ses propres réflexions morales sur le personnage de Mentor du Télémaque de Fénelon (A. Cheng).

Pour finir, on rappellera qu'aujourd'hui le mentor peut être désigné par les termes maître, conseiller, père, guide, tuteur, moniteur, entraîneur, initiateur, voire coach ou gourou. Catherine Halpern dans sa critique de l'ouvrage de Françoise Waquet parue dans Libération, souligne le lourd héritage du mot maître, que l'on hésite à utiliser de nos jours : « Ah, les vilains mots qui semblent dire l'autorité et la soumission, la duplication et le refus de l'innovation, mais aussi l'exclusion et les basses manœuvres $\rangle^{52}$. Le maître est mort ; vive le mentor !

\footnotetext{
${ }^{52}$ Catherine Halpern, «Le disciple et la voie de son maître », Libération, 25 septembre 2008.

https://journals.uvic.ca/index.php/sator/index
} 